\title{
Adaptive Traction Drive Control Algorithm for Electrical Energy Consumption Minimisation of Autonomous Unmanned Aerial Vehicle
}

\author{
Aleksandr Korneyev* (Doctoral student, Riga Technical University, Riga, Latvia), \\ Mikhail Gorobetz (Associate Professor, Riga Technical University, Riga, Latvia), \\ Ivars Alps (Researcher, Riga Technical University, Riga, Latvia), \\ Leonids Ribickis (Professor, Riga Technical University, Riga, Latvia)
}

\begin{abstract}
The paper aims at researching and developing an adaptive control system algorithm and its implementation and integration in the control system of the existing unmanned aerial vehicle (UAV). The authors describe the mathematical model of UAV and target function for energy consumption minimisation and possible searching algorithms for UAV optimal control from an energy efficiency perspective. There are two main goals: to minimise energy consumption and to develop and investigate an adaptive control algorithm for UAV traction drive in order to increase energy efficiency.

The optimal control algorithm is based on two target function values, when comparing and generating corresponding control signals. The main advantage of the proposed algorithm is its unification and usability in any electrical UAV with a different number of traction drives, different or variable mass and other configuration differences without any initial manual setup. Any electric UAV is able to move with maximal energy efficiency using the proposed algorithm.
\end{abstract}

Keywords - Adaptive algorithms; Energy consumption; Unmanned aerial vehicles.

\section{INTRODUCTION}

Since the unmanned aerial vehicles (UAVs) are increasingly used in various fields of human activity, their useful characteristics have become topical, and the flight distance is one of those. The battery capacity, the total mass, the efficiency of engines and converters (ESC regulators), control modes, as well as external influences (e.g., wind and temperature) affect the flight range of the UAV. As it is not possible to change external influences, it is necessary to adapt to them. It is required to build a control system that will adapt to changing external parameters and in the case of cargo delivery it will also react to total weight of the UAV changes after delivery, in order to find the control mode for optimal energy consumption for most efficient use of the battery. Most studies devoted to adaptive UAV systems investigate the stability of the aircraft.

Paper [1] presents the UAV usage options to fulfil power engineering tasks, improvements and their explanation. Paper [2] proposes the real-time problem solution for operational planning to minimise energy consumption of the UAV for the routes with obstacles. Euclidean calculations are compared by energy consumption criteria in [3]. Energy efficient optimisation algorithm for UAV charging network is described in paper [4]. UAV cooperation in network is described in [5] and [6]. Non-linear model forecasting strategy of UAV trajectory detection is proposed in [7], while energy efficient communication and UAV trajectory optimisation are discussed in [3]. Energy-saving for unmanned aerial vehicles is considered in [8], where a compromise between the stable operation of system and energy consumption is applied, so that the decrease in energy consumption does not reduce the quality of the whole system. In [9], planning a UAV optimal trajectory route total energy consumption 72.3397 Wh was achieved using an adaptive neural network control method, while without using this method, the average energy consumption was $96.593 \mathrm{Wh}$.

The optimisation of energy efficiency for small cellular networks using a coordinated UAV network was considered in [10], considering the coordination of small air cells. The energy efficiency maximising problem was formulated and presented in the non-convex fractional optimisation form. Such a problem is quite difficult to solve directly; therefore, it is divided into two subtasks and effectively solved by the proposed iterative algorithm. Path generation for UAVs with minimal energy consumption is proposed by the authors of [11]. In the present article, the paths of minimum energy between the predetermined initial and final configuration of the quadrotor are determined by solving the optimal control problem with respect to angular accelerations of four propellers. This theory is illustrated in several scenarios for the DJI Phantom 2 quadrotor. With the same UAV initial parameters, it is logical to assume that a short path consumes less energy than a longer one. The authors propose a new strategy for calculating the minimum time trajectories for quadrotors in a bounded environment. The transverse coordinates expressing the distance from the frame path are used to parameterise the position of the vehicle, and the spatial parameter is used as an independent variable. The strategy is proven by numerical calculations using two different illustrative scenarios [12].

The background analysis allows concluding that the minimisation of energy consumption of an unmanned aerial vehicle is a topical issue.

\section{PROBLEM FORMULATION}

The main practical goal of the research is to minimise energy consumption of UAV. The main scientific goal of the research

* Corresponding author.

E-mail: Aleksandrs.Kornejevs_1@ @rtu.lv

(C)2019 Aleksandr Korneyev, Mikhail Gorobetz, Ivars Alps, Leonids Ribickis.

This is an open access article licensed under the Creative Commons Attribution License

(http://creativecommons.org/licenses/by/4.0), in the manner agreed with Sciendo. 
is to study and to develop an adaptive control algorithm for UAV traction drive in order to increase energy efficiency of UAV.

The main research object is an adaptive algorithm that could be applied for various UAVs without initial settings, e.g., mass, engine speed, in order to move as efficiently as possible. The computer simulation of an adaptive quadcopter control algorithm is the final stage of the present research.

The main tasks of research are as follows:

- to study mechanical and electrical characteristics and to develop the mathematical model and target function for electrical energy consumption minimisation;

- to develop an optimal control algorithm;

- to create the UAV testbench model in order to obtain the real traction drive parameters;

- to develop the computer model with an adaptive algorithm and control system with adaptive controller of UAV take-off;

- to simulate motion of the UAV and to evaluate the workability of the control algorithm for different UAV configurations in real-time;

- to evaluate the efficiency of the selected adaptive searching algorithms.

\section{DEVELOPED STRUCTURE OF UAV CONTROL}

The adaptive optimisation algorithm is implemented on a microcontroller that is embedded in the existing UAV control and monitoring system. Modelling, estimation, and control for multirotor aerial vehicles, with a particular focus on the most common form - the quadrotor, have been investigated in [13]. The modifiable UAV quadcopter consists of a flying controller, brushless permanent magnet DC motor, electronic speed controllers and battery, voltage regulator (for low-voltage equipment powering), radio receiver, GPS module and built-in sensors. The optimisation controller consists of a microcontroller and an additional group of sensors, voltmeter, current meter (Hall sensor), accelerometer, barometric altimeter and GPS module. The optimisation controller is installed in the circuit between the master gear controller (coordinates) and the flight controller. The UAV flight controller has standard inputs: $A$ - aileron, $E$ - elevator, $T$ - throttle and $R$ - rudder. Instead of remote control, the optimisation controller is connected to the flight controller and plays the role of the UAV operator, which controls the UAV using the basic signals $\langle A, E, T, R\rangle$.

The improved control structure of quadcopter is demonstrated in Fig. 1.

Figure 2 shows a principal electrical scheme of the optimisation controller. The control signals $A, E, T$, and $R$ are fed to the Rx5 input of the optimisation controller.

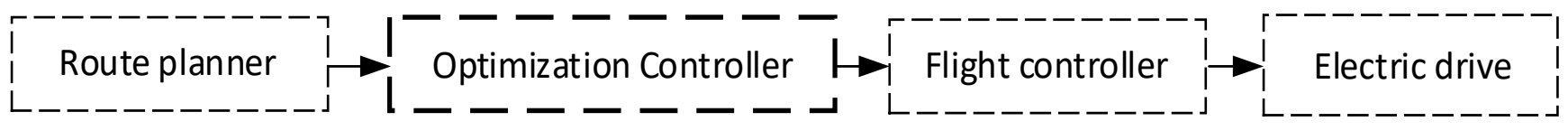

Fig. 1. Improved control structure of quadcopter UAV as example with optimization controller.

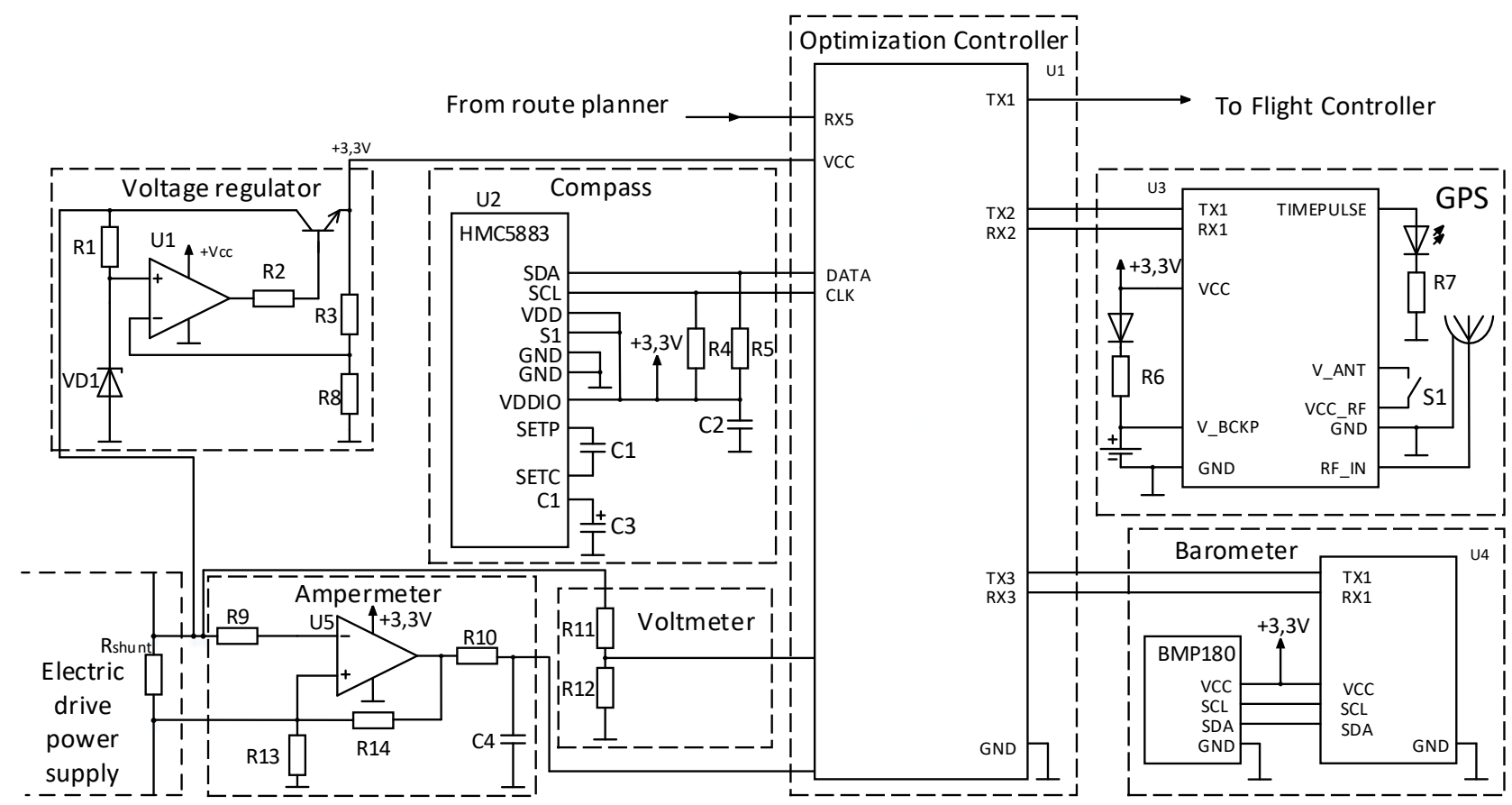

Fig. 2. Principal electrical scheme of optimisation controller.

The GPS module, compass, and barometer provide the optimisation controller with data about UAV's position relative to the destination and permissible deviations from the given route trajectory. The values of UAV's current and voltage getting to the input of the optimisation controller are converted to power consumption. To power the circuit element, on-board 
voltage is stabilised by a voltage regulator. Incoming data are processed according to a given optimisation algorithm and, as a result, the optimised control signals appear at the output of TX1.

\section{MATHEMATICAL MODEL OF UAV}

The model is based on the fundamental law of classical mechanics, actually Newton's second law of motion:

$$
F_{\text {vil }}-F_{\text {gr }}-F_{\text {ga }}=m a,
$$

where

$F_{\text {vil }}-$ UAV resultant force, $\mathrm{N}$;

$F_{\text {ga }}-$ air resistance force, $\mathrm{N}$;

$F_{\text {gr }}$ - gravity force, $\mathrm{N}$

$m$ - UAV mass, $\mathrm{kg}$;

$a-\mathrm{UAV}$ acceleration, $\mathrm{m} / \mathrm{s}^{2}$.

The following functional dependencies are defined for UAV velocity limits:

Max vertical velocity:

$$
\begin{gathered}
V_{\text {ver }}=\sqrt{\frac{2 m \cdot g_{0}}{\rho \cdot C_{\mathrm{D}} \cdot A_{\mathrm{eff}}} \sqrt{(F R-1)}}, \\
F R=\frac{F}{m \cdot g_{0}},
\end{gathered}
$$

where

$F$ - motor summary traction power, N;

$g_{0}$ - gravity acceleration value $9.81 \mathrm{~m} / \mathrm{s}^{2}$;

$C_{\mathrm{D}}$ - aerodynamic flow factor;

$A_{\text {eff }}$ - UAV effective area, $\mathrm{m}^{2}$;

$\rho-$ air density, $\mathrm{kg} / \mathrm{m}^{3}$;

$$
\begin{gathered}
F_{\mathrm{ga}}=\rho \cdot C_{\mathrm{D}} \cdot A_{\mathrm{eff}} \cdot v^{2}, \\
F_{\mathrm{gr}}=m \cdot g_{0} .
\end{gathered}
$$

UAV acceleration is calculated from the force equation:

$$
a=\left(F_{\mathrm{vil}}-F_{\mathrm{gr}}-F_{\mathrm{ga}}\right) / m .
$$

UAV velocity:

$$
v=\int_{0}^{\tau} a d t .
$$

UAV distance:

$$
s=\int_{0}^{\tau} v d t .
$$

Mechanical power (instantaneous):

$$
N_{(t)}=F_{t} \cdot v(t) \text {. }
$$

Mechanical work:

$$
A=\int_{0}^{\tau} N \mathrm{~d} t / 3600
$$

Power consumption, $\mathrm{W}$ :

$$
P=U I
$$

Torque on the motor shaft, $\mathrm{Nm}$ :

$$
M_{\mathrm{sl}}=9950 \frac{P}{n} \text {. }
$$

Consumed energy from source:

$$
E=\int_{0}^{\tau} P d t / 3600
$$

\section{TARGET FUNCTION FOR ENERGY CONSUMPTION MINIMISATION}

The primary criterion of the target function is UAV energy consumption minimisation, which in general form is represented as the following equation:

$$
E=f_{1}(C, Q) \rightarrow \min
$$

The secondary criterion is a minimisation of the manoeuvre time that should be minimal in case when the primary criterion is satisfied, i.e., if more than one solution exists with the same minimal energy consumption, then manoeuvre with the shortest time should be selected:

$$
\tau=f_{2}(C) \rightarrow \min ,
$$

where

$E$ - electrical energy consumption for the manoeuvre completion, Ws;

$C$ - a set of adaptive control parameters, i.e., $C=(R, P, T, Y)$, where

$A$ - a roll control signal level, $\mu$ s - moving right, left;

$E$ - a pitch control signal level, $\mu$ s - moving back, forward;

$T$ - a throttle control signal level, $\mu \mathrm{s}$ - moving up, down;

$R$ - a yaw control signal level, $\mu$ s - turn signal;

$Q$ - an uncontrollable parameter set, internal, external and environmental impacts;

$\tau$ - time spent for manoeuvre, s.

Start parameters: $A=0, E=0, T=0, R=0, a z(R) 0^{\circ}$, $\operatorname{targ}=0+\mathrm{n}^{\circ}$.

$$
\left\{\begin{array}{c}
E_{v}=\int I U \mathrm{~d} t=f(A, E, T, R, t) \rightarrow \min \\
\mid a z(R)-\text { targ } \mid \rightarrow 0 \\
\left|x(A, E)-x_{M}\right| \rightarrow 0 \\
\left|y(A, E)-y_{M}\right| \rightarrow 0 \\
\left|z(T)-\mathrm{z}_{M}\right| \rightarrow 0 \\
1000 \leq A \leq 2000 \\
1000 \leq E \leq 2000 \\
1000 \leq T \leq 2000 \\
1000 \leq R \leq 2000
\end{array}\right.
$$


The control signals are limited within minimal and maximal acceptable boundaries $a$ and $b$ :

$$
\left\{\begin{array}{l}
a_{R} \geq R \geq b_{R} \\
a_{P} \geq P \geq b_{P} \\
a_{T} \geq T \geq b_{T} \\
a_{Y} \geq Y \geq b_{Y}
\end{array} .\right.
$$

In the present research, the manoeuvre means the achievement of the target point $\mathrm{M}\left(x_{\mathrm{M}}, y_{\mathrm{M}}, z_{\mathrm{M}}\right)$ from the current UAV location $\mathrm{S}\left(x_{0}, y_{0}, z_{0}\right)$, within the condition:

$$
\left\{\begin{array}{l}
\left|x_{M}-x_{0}\right| \leq \varepsilon_{X} \\
\left|y_{M}-y_{0}\right| \leq \varepsilon_{Y} \\
\left|z_{M}-z_{0}\right| \leq \varepsilon_{Z}
\end{array},\right.
$$

where $\varepsilon_{X}, \varepsilon_{Y}, \varepsilon_{Z}$ - an acceptable precision by $\mathrm{O}_{x}, \mathrm{O}_{y}, \mathrm{O}_{z}$ axes. Energy consumption of UAV in continuous form is the following:

$$
E_{v}=\int_{0}^{\tau} \sum_{j=1}^{N} u_{j}(t, c(t)) \cdot i_{j}(t, c(t)) \cdot \mathrm{d} t,
$$

where

$E_{v}$ - consumed energy by traction drives for manoeuvre, Ws;

$t$ - momentary time values, $\mathrm{s}$;

$\tau$ - manoeuvre completion time, $\mathrm{s}$;

$N$ - a number of UAV traction drives (traction motor and electronic speed controllers);

$j-$ an index of UAV traction drive;

$u_{j}(t)$ - a momentary voltage value of $j$-th traction drive, $\mathrm{V}$;

$i_{j}(t)$ - a momentary current value of $j$-th traction drive, A;

$c(t)$ - a set of momentary control signal values, $\mu$ s.

Improving energy efficiency to reduce electrical energy consumption was investigated in [14].

\section{ALGORITHM FOR OPTIMAL UAV REAL-TIME CONTROL}

\section{A. Halving Algorithm}

In the present paper, the real-time modification of the uniform search algorithm is developed as an optimal search algorithm.

The task of the algorithm is to find minimum of the unimodal function $E(c)$, where $c-$ a control signal (absolute in $\%$, or relative in $\mu s)$, which is defined in a closed range of acceptable values $D=[\mathrm{a}, \mathrm{b}]$,

$$
\min E(C)=E\left(c^{*}\right), c c[a, b] .
$$

Halving algorithm or even dichotomous searching algorithm attempts are in pairs. The coordinates of each pair are different from the other pair for the amount

$$
\delta_{c}<\varepsilon_{c},
$$

where $\varepsilon_{c}-$ the required solution accuracy.

Attempts are made in the range of values of the control signal (traction force value range TFVR). After the function $E(c)$ values are obtained, several TFVR are eliminated from search due to unimodality. Value $\delta_{c}$ is detected with the required accuracy. This algorithm belongs to a class of successive searching.

Algorithm mathematical presentation is the following:

1. Assignment $r=1, a^{1}=a, b^{1}=b$,

$$
T F V R_{1}=\left[a^{1}, b^{1}\right] \text {. }
$$

2. Value calculation (see Fig. 3)

$$
c_{0}^{r}=\frac{a^{r}-b^{r}}{2}, c_{1}^{r}=c_{0}^{r}-\frac{\delta_{c}}{2}, c_{2}^{r}=c_{0}^{r}+\frac{\delta_{c}}{2} .
$$

3. Read the function $E(c)$ values according to wattmeter measurements $E\left(c_{1}^{r}\right), E\left(c_{2}^{r}\right)$.

4. If $E\left(c_{1}^{r}\right)<E\left(c_{2}^{r}\right)$, then perform assignments

$$
a^{r+1}=a^{r}, b^{r+1}=c_{0}^{r}, T F V R_{r+1}=\left[a^{r+1}, b^{r+1}\right]
$$

or perform assignments

$$
a^{r+1}=c_{0}^{r}, a^{r}, b^{r+1}=b^{r}, T F V R_{r+1}=\left[a^{r+1}, b^{r+1}\right] .
$$

5. If $\left|T F V R_{r+1}\right| \leq \varepsilon_{c}$, then END,

or perform $r=r+1$ and go to Step 2 .

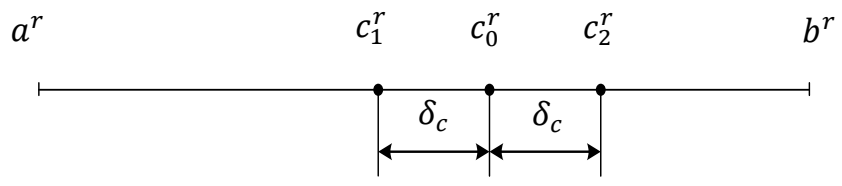

Fig. 3. Calculation of $c_{0}^{r}, c_{1}^{r}, c_{3}^{r}$ values.

Under the same conditions, any point from the current uncertainty interval may be accepted as the approximated minimum point $c^{*}$. The uniform halving algorithm is described in Fig. 4.

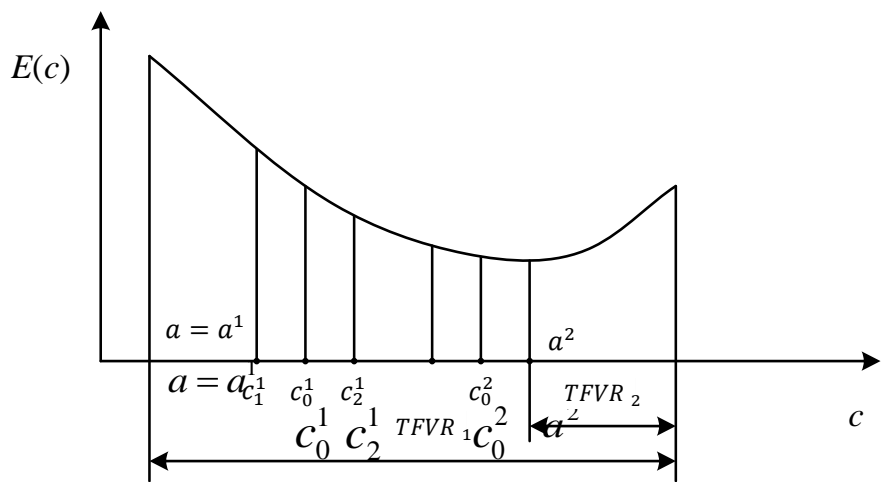

Fig. 4. The first two iterations of a one-dimensional unimodal function $E(c)$ with a halving algorithm.

The TFVR decrease twice after each iteration. Since in each iteration only two measurements are required, this algorithm can be accepted as applicable to the optimal management of UAV. 


\section{B. General UAV Control Algorithm}

In the present paper, the searching for optimal control methods are based on comparing the values of two target functions and making the respective decisions due to the improvement (success) or deterioration (failure).

The UAV optimal control algorithm in general form is the following.

1. Increase the control signal until starting.

2. Start control signal generation - set the control signal value.

3. Measurement - Measure or calculate the value of the target function.

4. Optimization - use a certain optimisation method to make a decision and perform an action on the next control signal value.

5. Checking for optimal value - If the optimum value is not reached, returned to Step 2. Use a new control signal until the optimal control value is reached, then go to Step 5.

6. Waiting - when the optimum value is reached, after a certain period it is necessary to recheck the optimum value because the movement conditions may change, then repeat from Step 2.

The developed algorithm for the UAV optimisation controller is the following.

Step 1. Initial control signal $c$ is set.

Step 2. By measuring the acceleration, the time moment is found when $a<$ eps $\approx 0$, so $v \approx$ const. Thus, the transient process is not considered.

Step 3. Current energy consumption value is stored as $\mathrm{e}_{0}$, time moment as $t_{0}$ and distance as $s_{0}$.

Step 4. After the time $t_{\mathrm{f}}=$ const, i.e., constant for any measurements, the relative energy consumption per meter is calculated as $\mathrm{d} e=\left(e-e_{0}\right) /\left(s-s_{0}\right), \mathrm{Wh} / \mathrm{m}$.

Step 5. If $\mathrm{d} e_{1}$ is not defined yet, then $\mathrm{d} e_{1}=\mathrm{d} e$, or $\mathrm{d} e_{2}=\mathrm{d} e$.

Step 6. If $\mathrm{d} e_{2}$ is defined, then go to Step 7 or $c=c+\mathrm{d} c$ and go to Step 2.

Step 7. If $\mathrm{d} e_{2}<\mathrm{d} e_{1}$, then

current control signal $c$ is better than the previous one, and the search is moving towards the extremum point, i.e., the search step is successful, and according to the optimal search algorithm, new $\mathrm{d} c_{\text {suc }}$ is set or calculated and next $c$ value is defined as $c=c+\mathrm{d} c_{\text {suc }}$

or

current control signal c is worse than the previous one, i.e., the search step is unsuccessful, and according to the optimal search algorithm, new $\mathrm{d} c_{\text {uns }}$ is set or calculated and next $x$ value is defined as $c=c+\mathrm{d} c_{\text {uns. }}$.

Step 8 . Check the optimality achievement conditions:

If conditions are satisfied, then

search is stopped,

optimal control $c^{*}=c$ and

next optimality check and search will be activated after the predefined delay $t_{\mathrm{g}}$.

or, go to Step 2.

\section{COLLECTION OF UAV REAL TRACTION DRIVE PARAMETERS}

For testing the algorithm, three different UAV motors have been selected: BR2216-KV810, BR2212-KV920 and BR2212KV980, which are usually used in quadcopters. The propeller 1045 is used, i.e., 10-inch diameter and 4.5-inch step.

The special testbench for UAV traction testing and modelling is developed. It allows evaluating the power characteristics of motor at different loads. It is also used to simulate the UAV motion. The measured values are accumulator battery current, voltage and traction force of the traction drive (motor with propeller and ESC).

The testing system is presented in Fig. 5. and the schematic of the motor testing part is shown in Fig. 6.

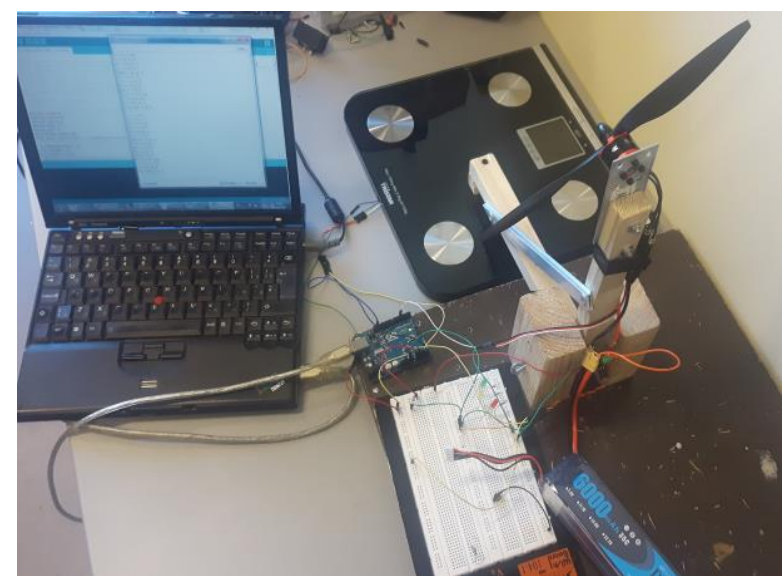

Fig. 5. Motor testing and UAV simulation system.

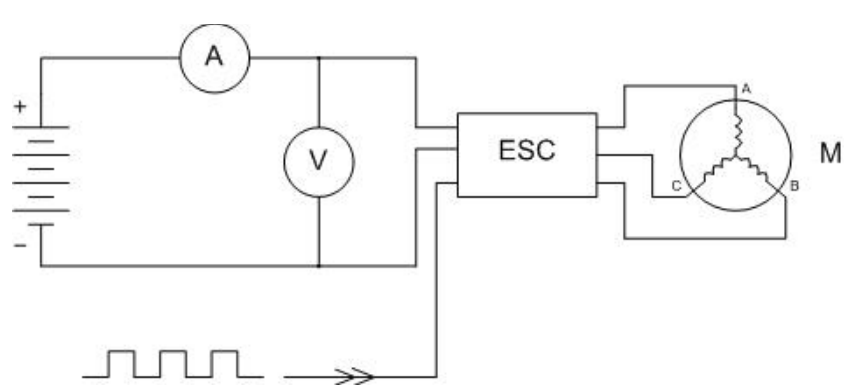

Fig. 6. Schematics of UAV traction drive testbench.

The motor is connected to the ESC and for control of the ESC the microcontroller board is used. It generates a pulse-position modulation signal with the duration between $1000 \mu \mathrm{s}$ and $2000 \mu \mathrm{s}$. The controller obtains the real battery voltage and current values, the motor and the propeller when receiving the control signal from the controller give the traction force measured by the load sensor module. The traction force is calculated using the equation $F=9.81 \mathrm{w}$ and motor power is calculated as $P_{\text {electric drive }}=U I$.

Since in real conditions the optimisation algorithm does not consider the elements of the electric drive separately but takes into account the total energy consumption, the losses in the wires and the losses in the speed controller are not calculated. Thus, the power consumed by the electric drive consists of

$$
P_{\text {electric drive }}=\Delta P_{\text {wire }}+\Delta P_{\mathrm{ESC}}+P_{\text {motor }},
$$


where

$\Delta P_{\text {wire }}$ - power loses in wires;

$\Delta P_{\mathrm{ESC}}-$ power loses in the speed converter;

$P_{\text {motor }}-$ motor power (including loses).

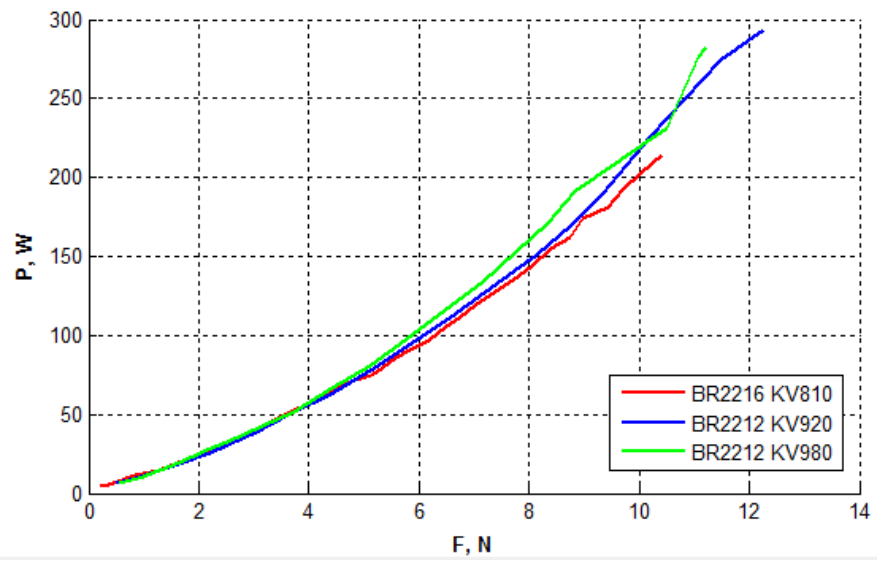

Fig. 7. Power-force curves of three motors.

The power graph for BR2216 KV810, BR2212 KV920, at various traction force values from Table I is shown in Fig. 7. Figure 7 demonstrates obvious non-linearity that proves the possibility for energy consumption optimisation.

TABLE I

MEASUREMENTS AND CALCULATIONS OF BR2216 KV810 MOTOR

\begin{tabular}{|c|c|c|c|c|c|}
\hline $\boldsymbol{\%}$ & $\boldsymbol{U}, \mathbf{V}$ & $\boldsymbol{I}, \mathbf{A}$ & $\boldsymbol{m}, \mathbf{k g}$ & $\begin{array}{c}\text { Calculated } \\
\boldsymbol{F}, \mathbf{N}\end{array}$ & $\begin{array}{c}\text { Calculated } \boldsymbol{P}, \\
\mathbf{W}\end{array}$ \\
\hline 10 & 16.27 & 0.3 & 0.028 & 0.27 & 4.88 \\
\hline 40 & 16.22 & 3.2 & 0.375 & 3.68 & 51.90 \\
\hline 60 & 16.14 & 6 & 0.63 & 6.18 & 96.84 \\
\hline 80 & 15.89 & 11.4 & 0.96 & 9.42 & 181.15 \\
\hline 86 & 15.76 & 13.6 & 1.06 & 10.40 & 214.34 \\
\hline
\end{tabular}

Quadcopter efficiency equation is the following:

$$
\eta=\frac{A}{E}
$$

where

$$
\begin{aligned}
& A=A_{0 \mathrm{sec}}+A_{0.1 \mathrm{sec}}+\ldots+A_{n \mathrm{sec}} \\
& E=E_{0 \mathrm{sec}}+E_{0.1 \mathrm{sec}}+\ldots+E_{n \mathrm{sec}}
\end{aligned}
$$

The numerical example of mechanical work, consumed energy and efficiency calculation is presented for quadcopter following the given parameters:

- Motor - BR2216-KV810;

- $\rho=1.22 \mathrm{~kg} / \mathrm{m}^{3}$;

$-\quad C_{\mathrm{D}}=1.05$;

- $\quad A_{\text {eff }}=0.25 \mathrm{~m}^{2}$

- $g=9.81 \mathrm{~m} / \mathrm{s}^{2}$;

- $\quad m=1.5 \mathrm{~kg}$;

$-\mathrm{d} t=0.1 \mathrm{~s}$

Example is shown for the following values of variables assuming these values are not changing:

- $\quad F_{\mathrm{t} 1}=7.16 \mathrm{~N}-$ traction force of one motor;

- $\quad P_{1}=133.63 \mathrm{~W}-$ power of one motor;

- $\quad F_{\mathrm{t}}=28.6 \mathrm{~N}-$ traction force of 4 motors;

$-\quad F_{\text {gr }}=14.7 \mathrm{~N}, \mathrm{P}=534.52 \mathrm{~W}$;

- $\quad E=0.01485 \mathrm{Wh}$ in each time interval.
TABLE II

THE PART OF 100 M VERTICAL INTEGRATED TAKE-OFF

\begin{tabular}{|c|c|c|c|c|c|}
\hline$t, \mathrm{~s}$ & 0 & 0.1 & $\ldots$. & 15.5 & 15.6 \\
\hline$F_{\mathrm{ga}}, N$ & 0 & 0.28 & $\ldots$. & 13.93 & 13.93 \\
\hline$F, \mathrm{~N}$ & 13.93 & 13.65 & $\ldots$. & 0 & 0 \\
\hline$A, \mathrm{~m} / \mathrm{s}^{2}$ & 0 & 9.28 & $\ldots$. & 0 & 0 \\
\hline$V, \mathrm{~m} / \mathrm{s}$ & 0 & 0.93 & $\ldots$. & 6.59 & 6.59 \\
\hline$S, \mathrm{~m}$ & 0 & 0.09 & $\ldots$. & 99.48 & 100.14 \\
\hline$N, \mathrm{~W}$ & 0 & 26.59 & $\ldots$. & 188.85 & 188.85 \\
\hline$A, \mathrm{Wh}$ & 0 & 0.00074 & $\ldots$. & 0.00525 & 0.00525 \\
\hline
\end{tabular}

$$
\begin{gathered}
\sum A=A_{0 \mathrm{sec}}+A_{0.1 \mathrm{sec}}+\ldots+A_{n \mathrm{sec}}= \\
0+0.00074+0.00146+\ldots+0.00525=0.79669 \mathrm{Wh} \\
\sum E=E_{0 \mathrm{sec}}+E_{0.1 \mathrm{sec}}+\ldots+E_{n \mathrm{sec}}= \\
0.01485+0.0148+\ldots+0.01485=2.316253 \mathrm{Wh} \\
\eta=\frac{\sum A}{\sum E}=\frac{0.79669}{2.316253}=0.344
\end{gathered}
$$

\section{PROGRAMMABLE COMPUTER MODEL OF UAV TAKE-OFF}

The part of quadcopter computer model code is presented in Fig. 8. The part describes the computer and optimisation controller interaction to assess the ability of the microcontroller to perform the optimal control.

The microcontroller ATMEL328P is equipped with an adaptive algorithm for searching for optimal power consumption, and microcontroller output data control the propeller-motor pair of the test bench. The computer program receives the signal from the sensor and simulates the flight, but only the vertical component of the flight at a given altitude $S_{t}$ is used.

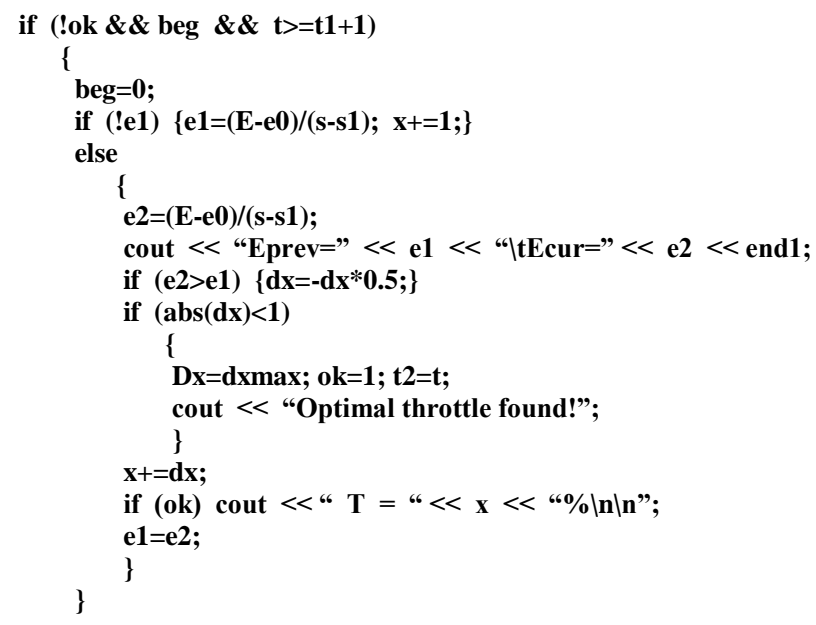

Fig. 8. The part of quadcopter computer code.

\section{Modelling Of UAV TAKe-OFF With AdAPTIVE ALGORITHM}

The experiment shows how the unified optimal algorithm performs the control of the UAV for the take-off motion. 

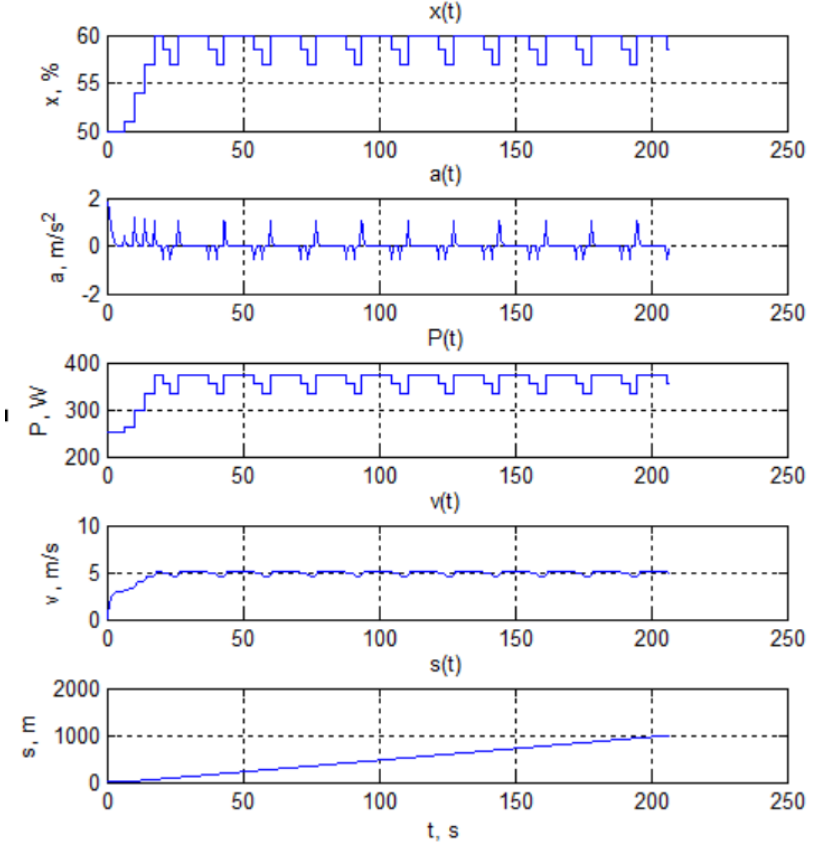

Fig. 9. Dynamics of the UAV with optimal control.

Figure 9 shows dynamics of five motion parameters of UAV - control signal $x(t)$, acceleration $a(t)$, power $P(t)$, velocity $v(t)$ and path $s(t)$. Initially, the algorithm increases the control signal until UAV starts the movement. Then the optimal control algorithm searches for the optimal control signal with minimal energy consumption. In Fig. 9, this signal is $60 \%$, i.e., $1600 \mu \mathrm{s}$. The acceleration dynamics shows that the decision making about the control signal change happens at the moment when the acceleration is close to zero, i.e., the transient process is skipped. When the optimal control value is found, the algorithm maintains the found throttle, but every 10 seconds it checks whether the optimality condition is satisfied. Thus, in case of internal, external or environmental parameter changes the algorithm will be able to find another optimal control signal to keep the minimal energy consumption in real time.

TABLE III

DIFFERENT CONFIGURATION PARAMETERS OF QUADCOPTERS

\begin{tabular}{|c|c|c|c|c|}
\hline $\boldsymbol{N}$ & Motor & $\boldsymbol{M}, \mathbf{k g}$ & $\boldsymbol{A}_{\text {eff }} \mathbf{m}^{2}$ & $\boldsymbol{C}_{\mathbf{D}}$ \\
\hline 1 & BR2216KV810 & 1.3 & 0.24 & 1.05 \\
\hline 2 & BR2216KV810 & 1.6 & 0.25 & 1.10 \\
\hline 3 & BR2216KV810 & 1.9 & 0.26 & 1.15 \\
\hline 4 & BR2216KV810 & 2.2 & 0.27 & 1.20 \\
\hline 5 & BR2212KV920 & 1.3 & 0.24 & 1.05 \\
\hline 6 & BR2212KV920 & 1.6 & 0.25 & 1.10 \\
\hline 7 & BR2212KV920 & 1.9 & 0.26 & 1.15 \\
\hline 8 & BR2212KV920 & 2.2 & 0.27 & 1.20 \\
\hline 9 & BR2212KV980 & 1.3 & 0.24 & 1.05 \\
\hline 10 & BR2212KV980 & 1.6 & 0.25 & 1.10 \\
\hline 11 & BR2212KV980 & 1.9 & 0.26 & 1.15 \\
\hline 12 & BR2212KV980 & 2.2 & 0.27 & 1.20 \\
\hline
\end{tabular}

In the present research, two quadcopters with different mass, area, drag rate values and with different motors are simulated in such a way. The different configuration parameters of quadcopters are shown in Table III.

Figures 10-13 provide the comparison of the UAV take-off simulation results when an optimal throttle signal is constant and known before and with the optimal control algorithm, when this value is unknown.

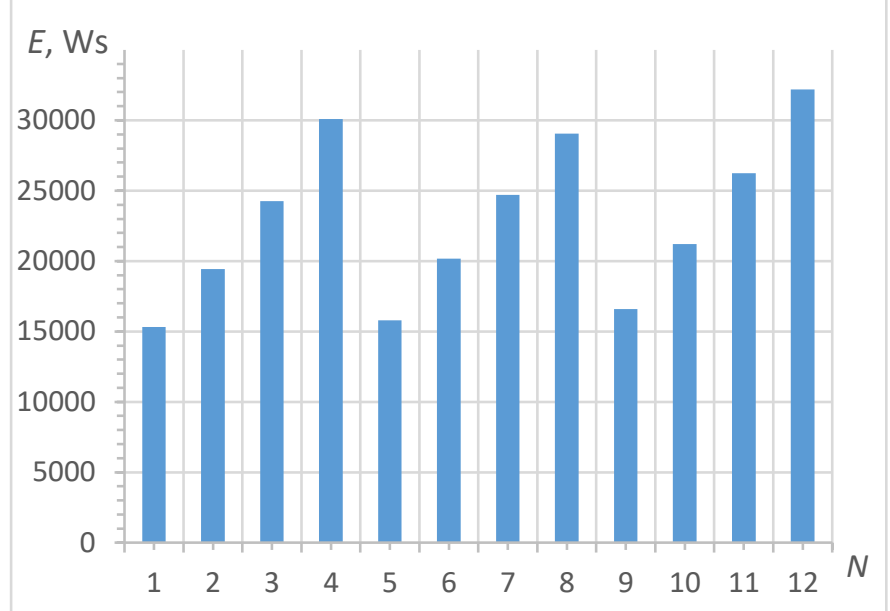

Fig. 10. Energy consumption for different quadcopters with an optimisation algorithm for $1000 \mathrm{~m}$ flight.

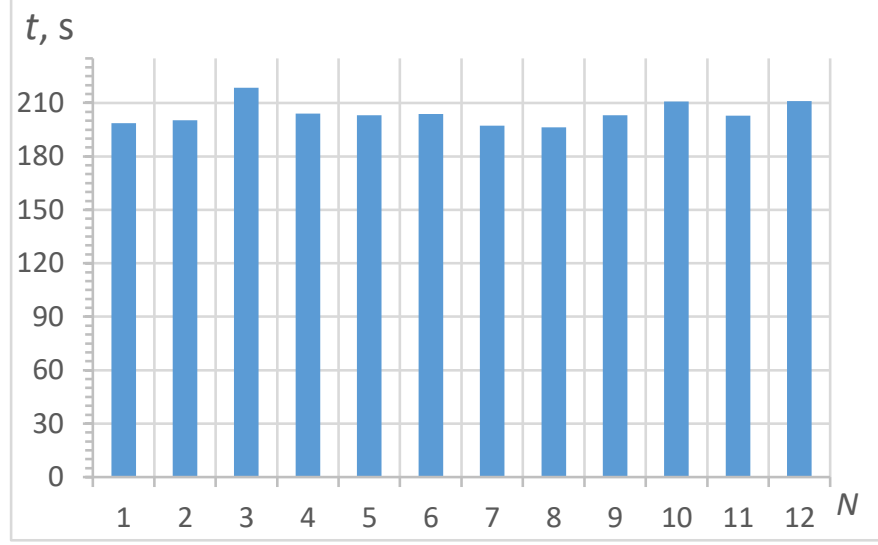

Fig. 11. Time spent for different quadcopters with an optimisation algorithm for $1000 \mathrm{~m}$ flight.

Results show that the algorithm is able to find an optimal solution in all cases. The search for an optimal solution needs time and it depends on the quadcopter mass. However, in practice, it is impossible to know the optimal value at the beginning of the control process due to different factors of the UAV and its environment. Therefore, it is necessary to adapt the control to these factors and minimise the energy consumption all the time during the flight. 


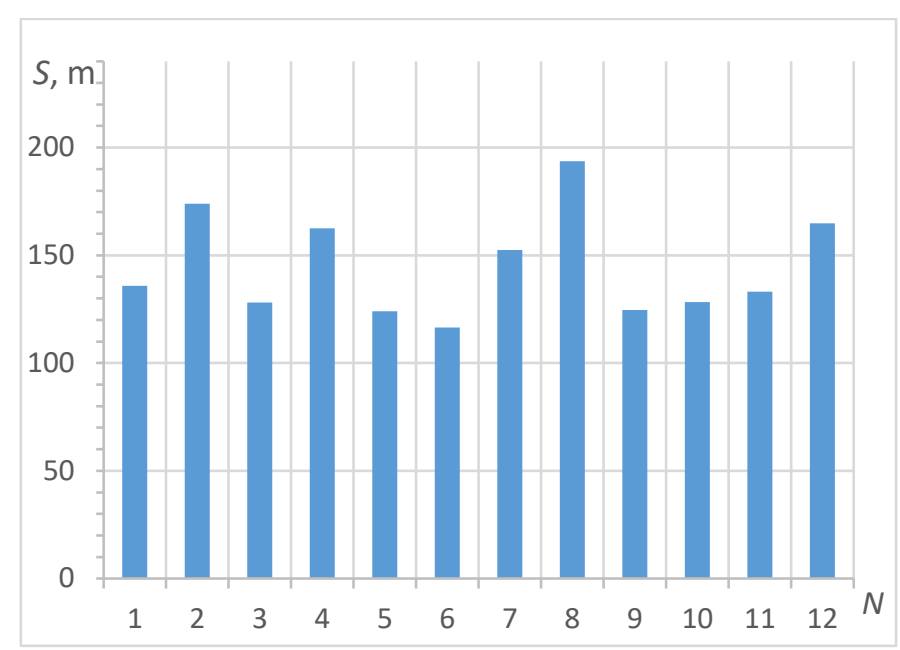

Fig. 12. The altitude at which the optimisation algorithm finds the optimal power consumption.

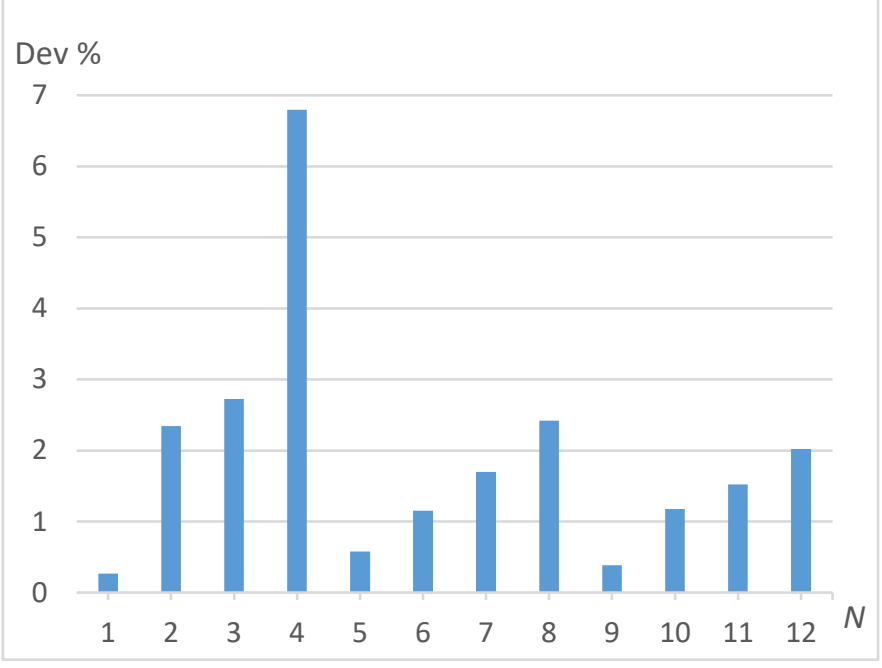

Fig. 13. The difference in power consumption when the optimisation algorithm is running.

\section{CONCLUSION}

The main tasks of research have been fully completed: UAV control system with adaptive controller has been developed; the target function of energy consumption has been defined, the optimal control algorithm has been created and successfully tested on the real traction drive testbench and the software model.

The developed optimisation algorithm generates the optimal control signals in the shortest time $-196.36 \mathrm{~s}$ and over the largest distance $-193.69 \mathrm{~m}$ with motor mass $2.2 \mathrm{~kg}$, effective area $0.27 \mathrm{~m}^{2}$ and drag rate 1.2 (configuration No. 8). The longest time $218.46 \mathrm{~s}$ till optimal control signals are reached is spent by the quadcopter with motor mass $1.9 \mathrm{~kg}$, effective area $0.26 \mathrm{~m}^{2}$ and drag rate 1.15 (configuration No. 3). The quadcopter with motor mass $1.6 \mathrm{~kg}$, effective area $0.25 \mathrm{~m}^{2}$ and drag rate 1.1 (configuration No. 6) travels the shortest distance until the optimal control signals are found $-116.43 \mathrm{~m}$. The lowest power consumption $15317.7 \mathrm{Ws}$ is provided with motor mass $1.3 \mathrm{~kg}$, effective area $0.24 \mathrm{~m}^{2}$ and drag rate 1.05 (configuration No. 1), but the highest power consumption $32178.7 \mathrm{Ws}$ is provided with motor mass $2.2 \mathrm{~kg}$, effective area
$0.27 \mathrm{~m}^{2}$ and drag rate 1.2 (configuration No. 12). It proves that the energy consumption to take-off at the altitude of $1000 \mathrm{~m}$ increases almost linearly as mass and area of the quadcopter increase independently of different motors, but the altitude when the algorithm finds the optimal control signal changes randomly. The smallest deviation of $0.26641356 \%$ from the optimal control signal is shown by the quadcopter with motor mass $1.3 \mathrm{~kg}$, effective area $0.24 \mathrm{~m}^{2}$ and drag rate 1.05 (configuration No. 1).

The proposed adaptive algorithm can claim for versatility and be used in various types of unmanned electric vehicles quadcopters, airplanes, trains, etc., as well as in man-driven vehicles in autopilot mode. The design feature of the optimisation controller requires minimal intervention in the vehicle control system.

The time to find the optimal energy-saving control signal using the proposed algorithm does not affect the total amount of energy consumption.

\section{REFERENCES}

[1] G. Strupka, and I. Raṇkis, "Investigation of Unmanned Aerial Vehicle Routes to Fulfill Power Line Management Tasks," in Chen J. (eds) Advances in Human Factors in Robots and Unmanned Systems. AHFE 2018. Advances in Intelligent Systems and Computing, vol. 784. Springer, Cham., 2019. https://doi.org/10.1007/978-3-319-94346-6_6

[2] N. Bezzo, et al., "Online planning for energy-efficient and disturbanceaware UAV operations," 2016 IEEE/RSJ International Conference on Intelligent Robots and Systems (IROS), Dec. 2016. https://doi.org/10.1109/IROS.2016.7759738

[3] J. T. Economou, G. Kladis, and A. Tsourdos, "UAV optimum energy assignment using Dijkstra's Algorithm," 2007 European Control Conference (ECC), IEEE Xplore, 26 March 2015. https://doi.org/10.23919/ECC.2007.7068353

[4] L. Li, J. Wu, Y. Xu, J. Che, and J. Liang, "Energy-controlled optimization algorithm for rechargeable unmanned aerial vehicle network," 2017 12th IEEE Conference on Industrial Electronics and Applications (ICIEA), Feb. 2018. https://doi.org/10.1109/ICIEA.2017.8283046

[5] H. Ghazzai, M. B. Ghorbel, A. Kassler, and J. Hossain, "Trajectory optimization for cooperative dual-band UAV swarms," in Proc. IEEE GLOBECOM, Abu Dhabi, UAE, Dec. 2018, pp. 1-7. https://doi.org/10.1109/GLOCOM.2018.8648087

[6] M. Chen, F. Dai, H. Wang, and L. Lei, "DFM: A distributed flocking model for UAV swarm networks," IEEE Access, vol. 6, pp. 69141-69150, 2018. https://doi.org/10.1109/ACCESS.2018.2880485

[7] Y. Liu, J. V. Schijndel, S. Longo, and E. C. Kerrigan, "UAV energy extraction with incomplete atmospheric data using MPC," IEEE Transactions on Aerospace and Electronic Systems, vol. 51, no. 2, April 2015, pp. 1203-1215. https://doi.org/10.1109/TAES.2014.130657

[8] Y. D. Liu, and L. Ziarek, "Toward Energy-Aware Programming for Unmanned Aerial Vehicles," 2017 IEEE/ACM 3rd International Workshop on Software Engineering for Smart Cyber-Physical Systems (SESCPS), Buenos Aires, 2017, pp. 30-33 https://doi.org/10.1109/SEsCPS.2017.8

[9] R.-J. Wai, and A. S. Prasetia, "Adaptive Neural Network Control and Optimal Path Planning of UAV Surveillance System with Energy Consumption Prediction," in IEEE Access, vol. 7, pp. 126137-126153, 2019. https://doi.org/10.1109/ACCESS.2019.2938273

[10] C. Liu, W. Feng, J. Wang, Y. Chen, and N. Ge, "Aerial Small Cells Using Coordinated Multiple UAVs: An Energy Efficiency Optimization Perspective," in IEEE Access, vol. 7, pp. 122838-122848, 2019. https://doi.org/10.1109/ACCESS.2019.2938256

[11] F. Morbidi, R. Cano, and D. Lara, "Minimum-energy path generation for a quadrotor UAV," 2016 IEEE International Conference on Robotics and Automation (ICRA), Stockholm, 2016, pp. 1492-1498. https://doi.org/10.1109/ICRA.2016.7487285

[12] S. Spedicato and G. Notarstefano, "Minimum-Time Trajectory Generation for Quadrotors in Constrained Environments," in IEEE Transactions on Control Systems Technology, vol. 26, no. 4, pp. 1335-1344, July 2018. https://doi.org/10.1109/TCST.2017.2709268 
[13] R. Mahony, V. Kumar and P. Corke, "Multirotor Aerial Vehicles: Modeling, Estimation, and Control of Quadrotor," in IEEE Robotics \& Automation Magazine, vol. 19, no. 3, pp. 20-32, Sept. 2012. https://doi.org/10.1109/MRA.2012.2206474

[14] P. Apse-Apsitis, A. Avotins, and R. Porins, "Industrial Greenhouse Electrical Power Monitoring Using Secure Internet-of-Things (IoT) Platform," 2018 IEEE 6th Workshop on Advances in Information, Electronic and Electrical Engineering (AIEEE 2018), pp 1-4. https://doi.org/10.1109/AIEEE.2018.8592307

Aleksandr Korneyev is a Doctoral student at the Institute of Industria Electronics and Electrical Engineering of Riga Technical University. He received the degrees of B.Sc. and M.Sc. in electrical engineering from Riga Technical University in 2016 and 2018, respectively. The main fields of scientific interest are unmanned aerial vehicle, evolutionary algorithm, and energy efficiency optimisation. He has several publications in proceedings of international conferences.

Address: Riga Technical University, Azenes Str. 12/1, Riga, LV-1048, Latvia. E-mail: Aleksandrs.Kornejevs_1@ @rtu.lv

Mikhail Gorobetz, Dr. sc. ing., Associate Professor and Leading Researcher a the Institute of Industrial Electronics and Electrical Engineering of Riga Technical University. Results of research activity are published in various international scientific proceedings and journals in the fields of adaptive control, neural networks, genetic algorithms, modelling and simulation of dynamic processes. He is a leader of various national projects and international projects. He is the author of many study books and patented inventions. Address: Riga Technical University, Azenes Str. 12/1, Riga, LV-1048, Latvia. E-mail: Mihails.Gorobecs@rtu.lv

ORCID iD: https://orcid.org/0000-0001-6633-1919
Ivars Alps received Dr. sc. ing. degree in 2012 from the Institute of Industrial Electronics and Electrical Engineering at Riga Technical University. The main fields of scientific interest are neural networks, artificial immune algorithm, scheduling theory, intelligent electric transport systems. He has several publications in proceedings of international conferences, as well as he is a coauthor of several patents.

Address: Riga Technical University, Azenes Str. 12/1, Riga, LV-1048, Latvia. E-mail: Ivars.Alps@rtu.lv

Leonids Ribickis received Dr. habil. sc. ing. degree in 1994 from Riga Technical University. He has been an Academician since 2003, a full Member of the Latvian Academy of Sciences since 2007, Rector of Riga Technical University, Head of the Institute of Industrial Electronics and Electrical Drives of the Faculty of Power and Electrical Engineering at Riga Technical University, 1999. His fields of interest include power electronics, electrical drives, electrical machines, process control systems. He was leading various national and international projects. He is the author of many patents, books and publications.

Address: Riga Technical University, Azenes Str. 12/1, Riga, LV-1048, Latvia. E-mail: Leonids.Ribickis@rtu.lv

ORCID iD: https://orcid.org/0000-0001-9077-198 